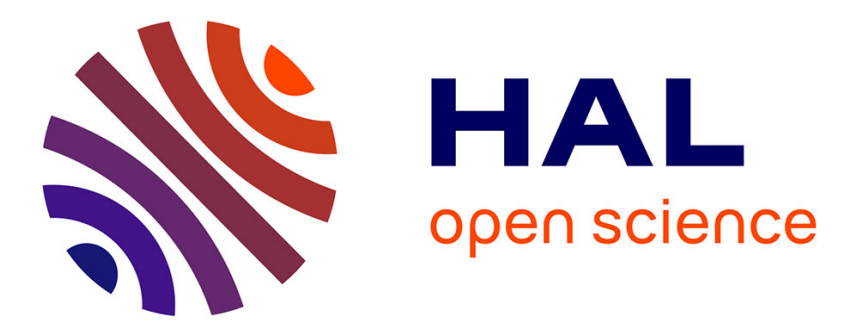

\title{
Online reaction monitoring by single-scan 2D NMR under flow conditions
}

\author{
Corentin Jacquemmoz, François Giraud, Jean-Nicolas Dumez
}

\section{To cite this version:}

Corentin Jacquemmoz, François Giraud, Jean-Nicolas Dumez. Online reaction monitoring by singlescan 2D NMR under flow conditions. Analyst, 2019, 10.1039/C9AN01758E . hal-02359999

\section{HAL Id: hal-02359999 \\ https://hal.science/hal-02359999}

Submitted on 12 Nov 2019

HAL is a multi-disciplinary open access archive for the deposit and dissemination of scientific research documents, whether they are published or not. The documents may come from teaching and research institutions in France or abroad, or from public or private research centers.
L'archive ouverte pluridisciplinaire $\mathbf{H A L}$, est destinée au dépôt et à la diffusion de documents scientifiques de niveau recherche, publiés ou non, émanant des établissements d'enseignement et de recherche français ou étrangers, des laboratoires publics ou privés. 


\title{
Online reaction monitoring by single-scan 2D NMR under flow conditions
}

Received 00th January 20xx, Accepted 00th January 20xx DOI: $10.1039 / x 0 x \times 00000 x$

\author{
Corentin Jacquemmoz ${ }^{\mathrm{a}}$, François Giraud ${ }^{\mathrm{b}}$ and Jean-Nicolas Dumez ${ }^{\mathrm{a}}$
}

ABSTRACT: Nuclear magnetic resonance (NMR) spectroscopy is a powerful tool for reaction monitoring. Several devices have been recently developed for online monitoring, using flow NMR, of batch reactions carried out in realistic experimental conditions in terms of, e.g., agitation and temperature. Here we show that time series of single-scan ultrafast 2D NMR (UF2DNMR) spectra can be collected to monitor solution mixtures that circulate in a flow unit. Fast multidimensional NMR methods have a demonstrated potential to provide kinetic and mechanistic information on reactions. UF2DNMR makes it possible to collect 2D data set in less than one second, but relies on spatial encoding (SPEN) that is sensitive to sample motion, thus making online monitoring challenging. We characterize interference between flow and spatial encoding and provide pulse-sequence- and hardware-based solutions. The resulting method is illustrated with the monitoring of a saponification reaction. UF2DNMR with a flow unit is a promising tool for the online monitoring of organic chemical reactions.

\section{Introduction}

Reaction monitoring is an important component of chemical synthesis. The concentrations of reactants, products and sideproducts, reaction kinetics, and the nature of intermediates are examples of information that are accessed with monitoring methods, which then contribute to the design, optimization and mechanistic understanding of chemical reactions. ${ }^{1-3}$ Nuclear magnetic resonance (NMR) spectroscopy is an efficient and widely used tool for reaction monitoring. Reaction progress can to some extent be monitored offline, by sampling periodically a fraction of the reaction mixture. NMR spectroscopy, being noninvasive and non-destructive, is also particularly suitable for real-time monitoring of chemical reactions.

An important question for real-time monitoring is whether reaction conditions are properly replicated in the context of the NMR measurement. Batch reactions can be carried out inside the NMR magnet, in a standard NMR tube. However magnetic stirring then cannot be used, leading to different agitation and heat transfer compared to a classic round-bottom flask, and potentially different kinetics. ${ }^{4}$ The range of reaction conditions that may be accessed inside the magnet, in terms, of, e.g., temperature, atmosphere, or sample illumination, is also more limited. Online reaction monitoring with flow NMR is a possible solution for real-time monitoring in a broad range of reaction conditions. ${ }^{5-8}$ In this approach, the batch reaction is carried out

\footnotetext{
a. CEISAM, CNRS UMR6230, Université de Nantes, 44300 Nantes, France b. ICSN, CNRS UPR2301, Univ. Paris Sud, Université Paris-Saclay, 91190 Gif sur Yvette, France

+ Footnotes relating to the title and/or authors should appear here.

Electronic Supplementary Information (ESI) available: Supplementary figures and pulse sequence code. See DOI: 10.1039/x0xx00000x
}

in an external flask, and a fraction of the solution volume is circulated through a capillary between the flask and the NMR magnet. Early implementations of flow NMR reaction monitoring relied on dedicated flow probes, initially designed for hyphenation with liquid chromatography. ${ }^{9-12}$ More recently, solutions based on a "flow cell" or "flow tube" that can be inserted into virtually any high-field, high-resolution NMR probes have been described and commercialized. ${ }^{13,14}$ These accessories are very useful for the analysis of batch chemical reactions with reaction times of a few minutes or more, in a wide range of reaction conditions. ${ }^{15-17}$ Reactions on shorter timescales require the use of stopped flow ${ }^{18-20}$ or rapidinjection devices, ${ }^{21-25}$ with which the reaction is again carried out inside the NMR magnet. For small-scale reactions carried out with microfluidic devices, miniaturized NMR devices have been developed for inline monitoring with good mass sensitivity. ${ }^{26,27}$

The power of monitoring methods depends on how much information can be extracted from the reaction mixture. Realtime NMR reaction monitoring most frequently relies on $1 \mathrm{D}$ NMR of abundant nuclei, mainly ${ }^{1} \mathrm{H},{ }^{31} \mathrm{P}$ and ${ }^{19} \mathrm{~F}$. 2D NMR is also in principle very useful in cases of spectral overlap in 1D spectra, and when structural information is sought after. However the classic implementation of 2D NMR requires experiment durations of 5-10 minutes or more. This sets constraints on the frequency with which spectra can be acquired, and also may lead to artefacts or " $t_{1}$ noise" as signals are evolving over time due to the chemical reaction. These limitations are one of the motivations for the development of a variety of fast 2D NMR methods, such as Hadamard encoding, ${ }^{28}$ Non Uniform Sampling, ${ }^{29-31}$ spectral aliasing, ${ }^{32}$ or accordion experiments, ${ }^{33,34}$ that have found applications in reaction monitoring. ${ }^{35-38}$ Ultrafast 2D NMR (UF2DNMR) is the fastest approach to record 
arbitrary 2D spectra. ${ }^{39-41}$ It is based on spatial parallelization of the indirect time dimension of 2D experiments, and makes it possible to obtain spectra in a single scan. The usefulness of UF2DNMR for reaction monitoring has been illustrated with mechanistic studies of organic chemical ${ }^{42-47}$ and electrochemical reactions, ${ }^{48}$ carried out in an NMR tube. It has also been used as an online detector in combination with a chromatography column and an HPLC system, ${ }^{49,50}$ with no chemical reaction. Online reaction monitoring with UF2DNMR has been described using a benchtop NMR system, ${ }^{51-53}$ but single-scan limits of detection in the molar range and low resolution limit the applicability of this approach.

In this article, we show that 2D NMR spectra can be recorded in a single scan for online reaction monitoring using a commercially available flow tube. Flow conditions make the acquisition of high quality UF2DNMR spectra more challenging, as it relies on spatial parallelisation, and we describe and address several sources of signal losses and repeatability issues. The resulting method is used to monitor a saponification reaction. Online reaction monitoring by single-scan 2D NMR under flow conditions has the potential to provide expanded kinetic and mechanistic information in chemical synthesis.

\section{EXPERIMENTAL SECTION}

Samples. Unreacting mixture 1 is composed of methanol (203 $\mu \mathrm{L})$, ethanol $(292 \mu \mathrm{L}), \mathrm{n}$-propanol $(376 \mu \mathrm{L})$ and 2-butanol (463 $\mu L)$ q.s. $25 \mathrm{~mL}$ of $\mathrm{H}_{2} \mathrm{O}$. The concentration is near $200 \mathrm{mM}$ for each compound. Unreacting mixture 2 is composed of methanol (81 $\mu \mathrm{L})$, ethanol $(116 \mu \mathrm{L}), \mathrm{n}$-propanol $(150 \mu \mathrm{L})$ and 2-butanol $(186 \mu \mathrm{L})$ q.s. $10 \mathrm{~mL}$ of acetonitrile. The concentration is near 200 $\mathrm{mM}$ for each compound. For the saponification reaction, ethyl acetate was purchased from Sigma Aldrich and was used without further purification.

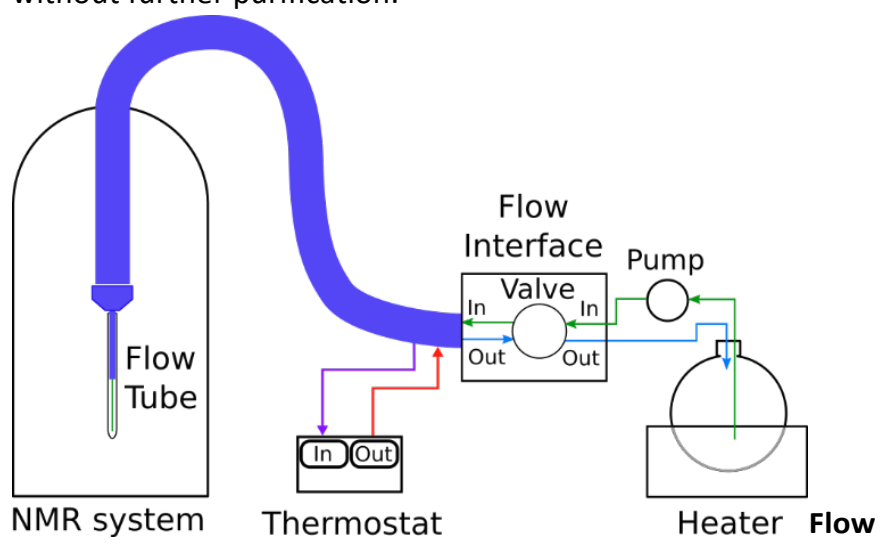

Figure 1: Schematic representation of the flow unit

NMR setup. A flow unit (Bruker InsightMR), schematized in Figure 1 is used to circulate a fraction of the solution between an external flask and the NMR tube. The solution goes through a capillary first to an HPLC piston pump (Knauer Azura 4.1S). The exit of the pump is connected to a valve used to either send the mixture to the NMR magnet (monitoring mode) or to send it back to the reacting flask (bypass mode). In monitoring mode, the solution flows through a capillary within a thermostatic and thermally insulated transfer line to the magnet, which can be heated to the desired temperature using a thermostatic water bath. Within the magnet, the solution flows through a modified NMR tube before returning to the reaction flask. A PEEK (PolyEther Ether Ketone) capillary of 0.5/0.8 mm inner/outer diameter is used here.

NMR experiments. All the experiments were carried out on a Bruker Avance III spectrometer operating at a ${ }^{1} \mathrm{H}$ Larmor frequency of $600.13 \mathrm{MHz}$ and equipped with a roomtemperature $5 \mathrm{~mm}$ TXI $1 \mathrm{H} / 13 \mathrm{C} / 15 \mathrm{~N}$ probe with triple-axis gradients and a GREAT $3 / 10-E$ gradient amplifier. The temperature regulation was set to $298 \mathrm{~K}$ for all experiments shown in the main text (experiments at other temperatures are described in the ESI). For all our experiments, Coherence Transfer Pathway (CTP) gradient and Spatial Encoding (SPEN) gradient were set on orthogonal axes. This is not mandatory, however, if CTP and SPEN gradient are along the same axis, peaks that originate from undesired CTP can accidentally appear in the SPEN dimension.

For UFCOSY experiments on unreacting mixtures in water, bipolar encoding gradients of \pm 0.0104 (for Z-axis) or \pm 0.008 (for $\mathrm{X}$-axis) $\mathrm{T} / \mathrm{m}$ were used in combination with $15 \mathrm{~ms}$ chirp pulses with $12 \mathrm{kHz}$ bandwidth. The acquisition consisted of a train of bipolar gradient pulses, of \pm 0.455 (for Z-axis encoding) or \pm 0.008 (X-axis encoding) $\mathrm{T} / \mathrm{m}$, with 128 loops and a duration of $256 \mu$ for each gradient pulse. A relaxation delay of $10 \mathrm{~s}$ was used including $5 \mathrm{~s}$ of presaturation. The excitation sculpting block was composed of a pair a $2 \mathrm{~ms}$ trapezoidal pulses and hard $\pi$ pulse surrounded by a pair of $1 \mathrm{~ms}$ gradient pulses (on $X \& Y$ axis for Z-SPEN experiments and on $Y$ axis for $X$-SPEN experiments).

For UFCOSY experiments on unreacting mixtures in acetonitrile, bipolar encoding gradients of \pm 0.010725 (for Z-axis encoding) or \pm 0.00825 (X-axis encoding) $\mathrm{T} / \mathrm{m}$ were used in combination with $15 \mathrm{~ms}$ chirp pulses with $12 \mathrm{kHz}$ bandwidth. The acquisition consisted of a train of bipolar gradient pulses of \pm 0.455 (for Zaxis) or \pm 0.35 (for X-axis) $\mathrm{T} / \mathrm{m}$, with 128 loops and a duration of $241 \mu \mathrm{s}$ for each gradient pulse. A relaxation delay of $10 \mathrm{~s}$ was used including $5 \mathrm{~s}$ of presaturation. The WET block was composed of four $20 \mathrm{~ms}$ sinc. 1 pulses with optimized power, each one followed by a $1 \mathrm{~ms}$ gradient pulse (on $X \& Y$ axis for Z-SPEN experiments and on $Y$ axis for $X-S P E N$ experiments).

For UFCOSY experiments on reacting mixtures shown in the main text, bipolar encoding gradients of \pm 0.0104 (for Z-axis encoding) or \pm 0.008 (X-axis encoding) $\mathrm{T} / \mathrm{m}$ were used in combination with $15 \mathrm{~ms}$ chirp pulses with $12 \mathrm{kHz}$ bandwidth. The acquisition consisted of a train bipolar gradient pulses of \pm 0.455 (for Z-axis) or \pm 0.35 (for $X$-axis) $\mathrm{T} / \mathrm{m}$ acquisition gradient, with 128 loops and a duration of $241 \mu$ s for each gradient pulse. A relaxation delay of $10 \mathrm{~s}$ was used including $5 \mathrm{~s}$ of presaturation. The excitation sculpting block was composed of a pair a $2 \mathrm{~ms}$ trapezoidal pulses and hard $\pi$ pulse surrounded by a pair of $1 \mathrm{~ms}$ gradient pulses (on $X \& Y$ axis for Z-SPEN experiments and on $Y$ axis for $X$-SPEN experiments).

Data processing. All processing was done using custom MATLAB code. The UF2DNMR data are imported in MATLAB then 
rearranged into a 2D matrix. Odd and even echoes are separated, and only odd echoes are processed here for simplicity. Along the spatial dimension, the data are inverse Fourier transformed, apodised with a Gaussian window, zerofilled and Fourier transformed. Along the spectral dimension the data are apodised with a sine window, zero-filled and Fourier transformed. Magnitude spectra are used in all cases. For build-up curves, the integration range of peaks were selected on the first spectrum and were used for integration on following spectra.

Online reaction monitoring. The saponification reaction was prepared according the following procedure. At room temperature, in a $100 \mathrm{~mL}$ flask with magnetic agitation, $20 \mathrm{~mL}$ of purified and degassed water were used to dissolve $125 \mu \mathrm{L}$ of $10 \mathrm{M}$ (1.25 eq / $62.5 \mathrm{mM}$ ) NaOH solution. The solution was fed to the flow tube with a flow rate of $3 \mathrm{~mL} / \mathrm{min}$ until the solution returned to the flask. With this setup, the dead time before the system equilibrate is about 1 minute and 40 seconds. This delay mainly depends on the flow rate and of the length of the transfer line (14 $\mathrm{m}$ in our case). A series of ultrafast acquisition were repeated every $10 \mathrm{~s}$ interlacing spatial encoding on $\mathrm{Z}$ and $\mathrm{X}$-axis. This delay is chosen to avoid any damage to the probe, and provides a repetition time that is still much shorter than with conventional (non-UF) experiments). $70 \mathrm{~s}$ after the beginning of the acquisitions, $100 \mu \mathrm{L}$ of ethyl acetate (1eq / 50 $\mathrm{mM}$ ) was introduced in the reaction flask. The acquisitions continued for about $35 \mathrm{~min}$.

\section{RESULTS AND DISCUSSION}

UF2DNMR of unreacting mixtures. Before using ultrafast 2D NMR for online reaction monitoring, the experiment needs to be implemented and validated on unreacting samples, the composition of which do not evolve in time. Limits of detection and repeatability are especially important in this context. While the theory and practice of UF2DNMR is well established on motionless sample, fewer examples exist for flowing samples. ${ }^{49,50}$ The results are expected to depend on the flow setup, and we focus here on a commercial version of a "flow unit" schematized in Fig. 1. ${ }^{13}$

UF2DNMR relies on spatial parallelization of the indirect time dimension of 2D NMR. This is achieved by the combined application of pulsed field gradients and frequency-swept chirp pulses, which induces a position-dependent evolution time. The information for all the virtual slices is obtained simultaneously during acquisition by echo planar spectroscopy imaging (EPSI ${ }^{54}$ ), which consists of a train of bipolar gradient pulses.

a shows the pulse sequence for the ultrafast COSY experiments, derived from the COSY pulse sequence by adding the spatial encoding and decoding elements. The pulse sequence also includes pulsed-field gradients for coherence selection and for solvent suppression.

When pulsed-field gradients are applied on a flowing sample, additional dephasing of the transverse spin magnetization occurs, compared to the case of a static sample. This can be compensated for using more complex gradient shapes, ${ }^{55,56}$ or taken advantage of to measure flow profiles. ${ }^{56,57}$ The combined application of a frequency swept pulses and magnetic-field gradients on a flowing sample is much less characterized. The results are expected to depend on whether a longitudinal $(z)$ or transverse (in the xy plane) axis is used for spatial encoding. Since standard high-resolution NMR probes are equipped with a $z$ gradient only, we first consider the case of longitudinal encoding.
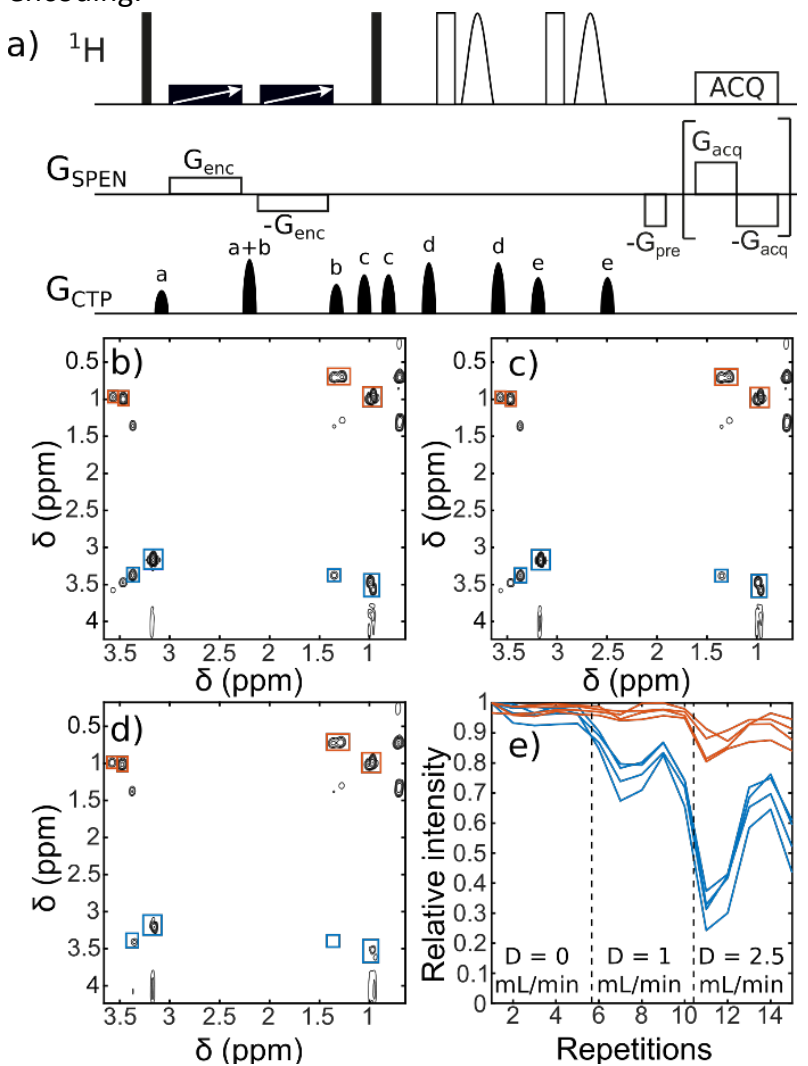

Figure 2: Pulse program used for ultrafast COSY acquisition with excitation sculpting for solvent suppression (a), ultrafast COSY spectra on model mixture 1 in water with flow rate of 0 (b), 1 (c) and $2.5 \mathrm{~mL} / \mathrm{min}$ (d) and integration variation as a function of repetitions at different flow rates (e). Black filled rectangles correspond to $90^{\circ}$ pulses and empty ones correspond to $180^{\circ}$ hard pulses. Black filled rectangles with arrows correspond to the $180^{\circ}$ adiabatic chirp pulses. All pulses have the same phase. For convenience CTP and SPEN gradients are displayed on different lines. They may be applied on orthogonal axis. Shaped pulses correspond to soft $180^{\circ}$ pulses used for excitation sculpting ( $2 \mathrm{~ms}$ trapezoidal pulses).

Figure $2 \mathrm{~b}-\mathrm{d}$ shows UFCOSY spectra acquired on a model mixture of short-chain alcohols in water in the flow tube, each in a single scan of $116 \mathrm{~ms}$, using longitudinal spatial encoding. The spectrum shown in Figure $2 \mathrm{~b}$ was obtained in the absence of flow, while the spectra of Figure 2c-d were obtained on a flowing sample, with flow values of 1.0 and $2.5 \mathrm{~mL} / \mathrm{min}$. It can already be noted that flow in this case does not lead to significant peak distortions or artefacts in the spectrum, so that good quality spectra are obtained in all cases. For the spectra acquired at $2.5 \mathrm{~mL} / \mathrm{min}$, the signal-to-noise ratio are in the 22 697 range with a median of 139 for the selected peaks. This would correspond to limits of detection of $\approx 5 \mathrm{mmol} / \mathrm{L}$.

It can also be noted in Figure $2 \mathrm{~b}$ - $\mathrm{d}$ that excellent solvent suppression is achieved for this experiment. While the effective 
acquisition bandwidth of the UFCOSY experiment, given by $1 /\left(2 T_{a}\right)$ where $T_{a}$ is the duration of a readout gradient, is of 3.01 ppm only, the filter bandwidth is much larger, to cover the frequency dispersion induced by the readout gradient. Unsuppressed solvent signals would thus fold into the spectrum. Solvent suppression was achieved here with presaturation combined with excitation sculpting ${ }^{58}$. In order to obtain repeatable solvent suppression longitudinal gradient should be avoided if possible. The use of gradient pulses with a $z$ component was found to result in poor repeatability for singlescan spectra, and this is independent of the use spatial encoding.

Figure $2 \mathrm{e}$ shows the volume of a selection of peaks in the UFCOSY spectrum, as a function of repetition number, concatenated for three different flow rates. Several features can be noted: $\mathrm{i} /$ the peak volume decrease on average when the flow rate increases; ii/ at a given flow rate, peak volumes vary by up to $50 \%$ when the experiment is repeated; iii/ features i/ and ii/ strongly depend on the chemical-shift offset in the indirect, spatially encoded dimension. With the parameters used here, peaks with higher chemical-shift value (in ppm) in the spatially encoded dimension suffer a more severe intensity loss. This effect was found to depend on the direction of the frequency sweep. If the chirp pulses frequency sweep from high to low chemical shifts, signals with higher chemical-shift values in the indirect dimension display the largest intensity losses (see Figure $2 \mathrm{~b}-\mathrm{e}$ ), whereas if the chirp pulses sweep from low to high chemical shifts, signals with lower chemical-shift values are most affected (see figure_SI 1).

A decrease of the peak volume as a function of the flow rate is expected because of an "outflow" effect: 8 a fraction of the sample that is excited by the first radiofrequency pulse exists the detection region of the coil before the start of acquisition (note that there is no inflow effect here, as the repetition time of $10 \mathrm{~s}$ is sufficient for near complete recovery of longitudinal magnetization). This is however not sufficient to account for the observed decrease. As the failure of excitation sculpting with $\mathrm{Z}$ gradients, the behaviour observed in Figure $2 \mathrm{e}$ is mainly due to interference between the spatial encoding process and sample motion, compounded by fluctuations of the flow profile.

Flow fluctuations. While signal losses may be tolerated to some extent when spatial encoding is used on a flowing sample, strong and uncontrolled fluctuations of peak volumes would be detrimental for monitoring applications. In order to characterize qualitatively the fluctuations, a simple gradient echo pulse sequence ${ }^{59}$, shown in Figure 3a, was used, with a repetition time of $55 \mathrm{~ms}$ and an echo time of $20.7 \mathrm{~ms}$. Figure 3c shows the fluctuations of the volume of the resulting 1D longitudinal image as a function of time (the "horns" at the edges of the image is due to the marked gradient non uniformity of triple axis gradient coils). ${ }^{60}$ Strong fluctuations are observed, which illustrate the pulsatile nature of the flow and its potentially deleterious effects. In this case the fluctuations had a frequency of about $2.2 \mathrm{~Hz}$, hence a period of $450 \mathrm{~ms}$ with a flow rate of $2.5 \mathrm{~mL} / \mathrm{min}$. The frequency varies with the flow rate, and could not be measured with sufficient accuracy to use a synchronized acquisition. Triggering the acquisition as a function of the flow rate, as in cardiac MRI, 61 was not feasible with our equipment.
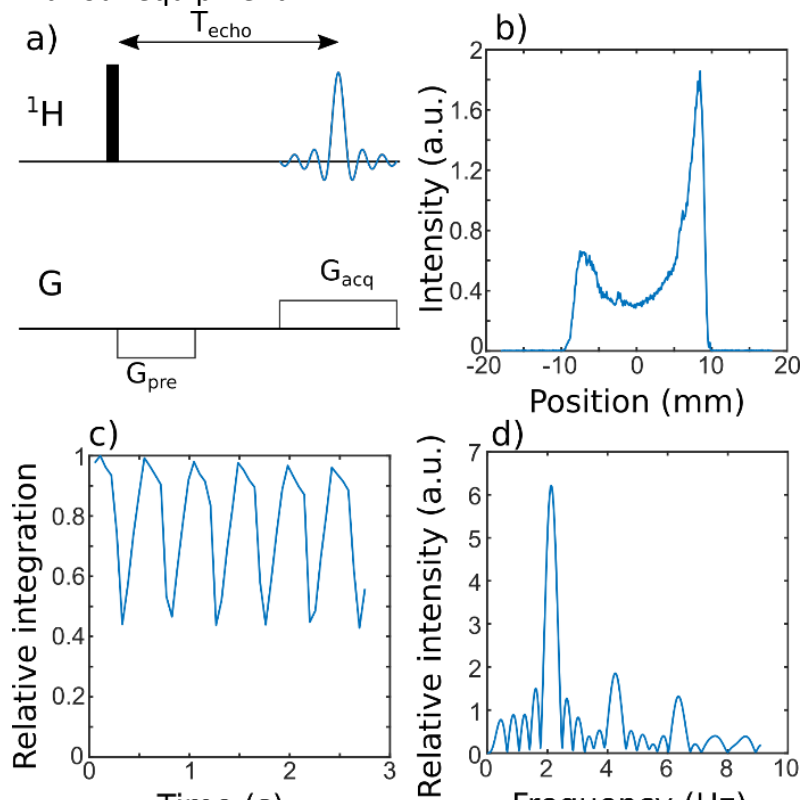

Frequency $(\mathrm{Hz})$

Figure 3: Gradient echo pulse sequence (a), image of the tube (b) filled with water obtained with $\mathrm{TE}=20.7 \mathrm{~ms}, \mathrm{TR}=55 \mathrm{~ms}$, and a flow rate of $2.5 \mathrm{~mL} / \mathrm{min}$. Variation of image integration as a function of time with a flow rate of $2.5 \mathrm{~mL} / \mathrm{min}$ (c) and Fourier transform of the signal (d).

The variation of integration shown in Figure $3 c$ is due to the use of a piston pump. In order to suppress these pulsations, the use of a peristaltic pump was considered, however this type of pump could not sustain the pressure necessary to flow the reaction mixture through the $14 \mathrm{~m}$ of capillary.

Note that the fraction of the sample located inside the capillary (green line in Fig. 1) through which the solution reaches the flow tube has a negligible contribution. Its volume is 60 times smaller than that of the rest of the sample, and more than half of this volume exits the coil region between excitation and detection.

Alternative encoding. As the spatial encoding method used previously was leading to important variations of peak intensities, the use of a second spatial encoding method, 62,63 that could be more robust against these effects, was studied. In this approach, shown in Fig. 4b, the two consecutive chirp pulses sweep in opposite directions and are separated by a hard $\pi$ pulse (Figure $4 b$ ). In order to have more precise information on the link between indirect chemical shift and flow rate, a sample consisting of the same short-chain alcohols was prepared in acetonitrile. This provides better distributed signals, as signals from $\mathrm{OH}$ groups are observed. The proximity of these signal to the solvent one, lead to the need to use a more selective solvent suppression method than excitation sculpting, and the WET (Water suppression Enhanced through $T_{1}$ effects) method $^{64}$ was chosen still used in combination with presaturation, resulting in the pulse sequences shown in Fig. 4. Carbon-13 decoupling was used during the WET sequence and acquisition to suppress solvent ${ }^{13} \mathrm{C}$ satellites. A series of UFCOSY 
repetitions were obtained at several flow rates with both spatial encoding blocks. Figure $4 \mathrm{~d}$ and $4 \mathrm{f}$ show the variation of peak volumes as a function of the flow rate for a selection of peaks. With chirp pulses that sweep in the same direction, intensity losses are maximum at one edge of the spectrum (which depends on the sweep direction) and minimum at the other edge. With chirp pulses that sweep in opposite directions, intensity losses are minimum at the centre of the spectrum and increase at the edges, so that the use of this encoding scheme mitigates in part the interference between flow and spatial encoding.

Note that no theoretical description is available, to the best of our knowledge, for the combined use of chirp pulses and magnetic field gradient under flow conditions; work on this aspect is in progress in our laboratory.

Interestingly, in contrast to what was seen with mixture 1 , in water (see Figure $4 \mathrm{c}-\mathrm{f}$ ) there are no strong variations of the peak volume from one spectrum to the next at a given flow rate for mixture 2, in acetonitrile. This effect is currently unexplained and further experiments with other solvents would help to analyse it.

Transverse encoding. When a triple-axis gradient probe is available, spatial encoding may also be used along a transverse axis. In the detected region of the sample, the flow is expected to be mostly laminar and in the longitudinal direction. There should thus be much less interference between spatial encoding and flow. Figure 5 shows a series of UFCOSY spectra obtained on the two model mixtures using spatial encoding along the $X$ axis of the probe. As previously, spectra were recorded several times at different flow rates. Transverse encoding leads to a loss of resolution in the spatially encoded dimension, because of the smaller length of the sample in that dimension, and of the limited gradient strength available. However, all of the undesirable features observed with longitudinal encoding disappear in this case: the intensity losses are much smaller than with longitudinal spatial encoding, there are no pulsations and the intensity losses do not seem to be linked to the chemical shift in indirect dimension. Interestingly, as seen on Figure $5 \mathrm{~b}$ and $\mathrm{d}$, the losses obtained with the acetonitrile solution are slightly more important than with water. a)

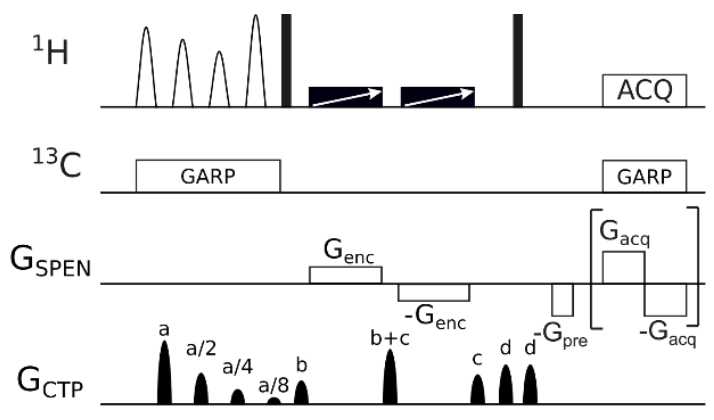

b)
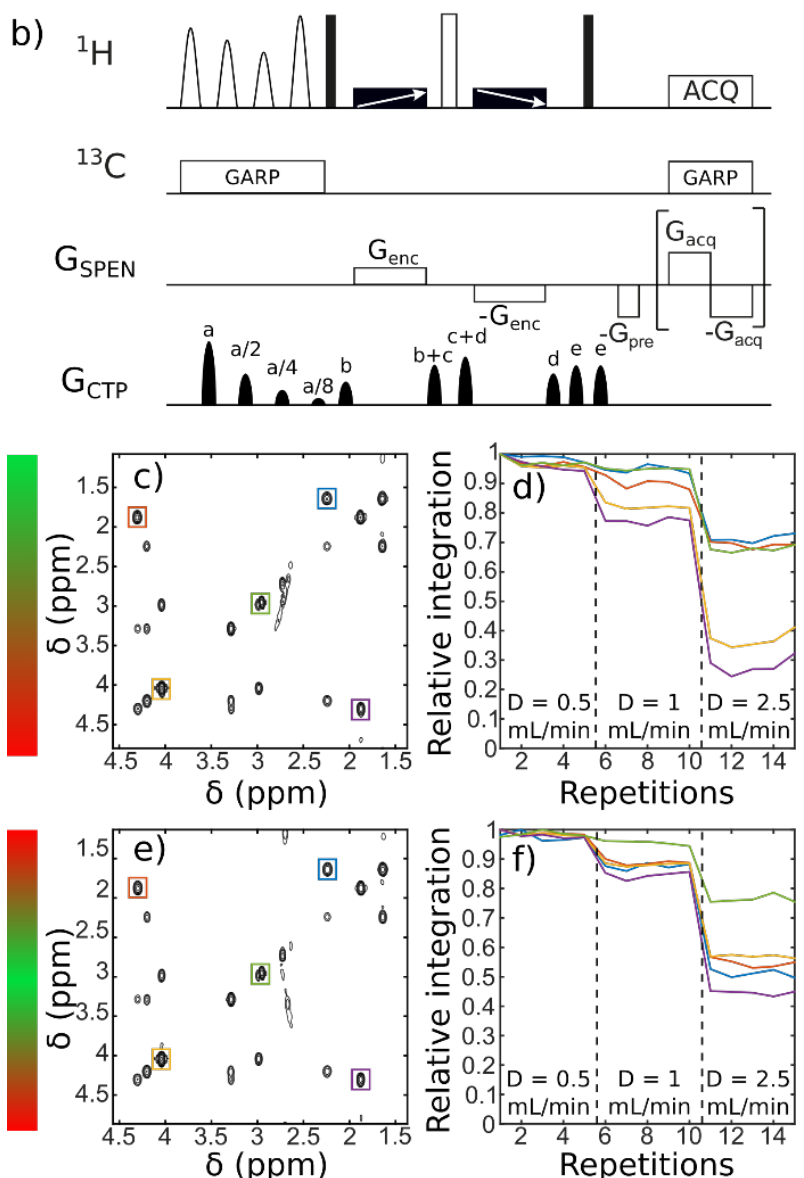

Figure 4: Pulse sequences for ultrafast COSY experiments with WET solvent suppression using "classical" (a) and "new" spatial encoding block. Ultrafast COSY spectra obtained on model mixture 1 in acetonitrile with classical (c) and new (e) spatial encoding and variation of peak integration as a function of repetitions at different flow rates ( $d$ and $f$ ). Red and green side bar illustrate how flow affects signals in the indirect dimension. Shaped pulses correspond to the pulses used for WET solvent suppression (20 ms Sinc1 pulses). The second, third and fourth pulses of the WET block are along $y$, while all other pulses are along $x$.

In this example of transverse encoding, the SNR is in the 911302 range with a median of 291 for a flow of $2.5 \mathrm{~mL} / \mathrm{min}$ the mixture in water. This would correspond to limits of detection of $\sim 2.5 \mathrm{mmol} / \mathrm{L}$.

It can be noted that the reported implementation of UF2DNMR for online monitoring on a benchtop spectrometer relied on transverse encoding, since the magnetic field gradient on the SpinsolveTM system is along a single transverse axis. In addition, the associated flow setup uses a peristaltic pump, and this together limits the occurrence and incidence of flow 
fluctuations. UF2DNMR on a flowing sample was also described in hyphenation with HPLC analysis, where the low flow rate 0.4 $\mathrm{mL} / \mathrm{min}$ ) did not lead to any problems, and with a custom chromatographic column. In the latter case a high flow rate 6 $\mathrm{mL} / \mathrm{min}$ ) was used, but eight scans were accumulated for phase cycling, so that potential fluctuations were smoothed. Moreover, pressured air was use to flow the mixture through the column, leading to a more continuous flow.

Reaction monitoring. In order to demonstrate feasibility of reaction monitoring using the Flow Unit in combination with UF2DNMR, a saponification reaction of ethyl acetate was studied. In order to compare longitudinal and transverse encoding, acquisitions with Z-axis and X-axis encoding were interlaced. Figure 6 shows examples of spectra obtained during the reaction and the evolution of several signals as a function of time. In both case, good quality spectra are obtained, with a time resolution that is much higher than what could be achieved with conventional 2D NMR. The use of transverse encoding is found to be important here to avoid signal fluctuations that would impede a kinetic analysis of the data. The possibility to measure rate constants from UF2DNMR data is illustrated in the ESI with a series of reactions carried out at three different temperatures. In the case of this simple reaction, the measurements can be validated by comparison with ${ }^{1} \mathrm{H}$ 1D NMR data.

Note that the delay between the introduction of the reactant in the reaction mixture and the apparition of signals on the spectra corresponds to the delay needed by the reaction mixture to reach the probe. This "dead time" leads to a loss of part of the information at the beginning of the reaction and is inherent to the use of the flow unit.

The saponification reaction was chosen here to illustrate the consequences of the choice of spatial encoding strategy on the quality of the UF2DNMR data in the case of a flowing sample. The relevance of UF2DNMR for reaction monitoring has been demonstrated for reactions carried out in an NMR tube, with the detection or assignment of intermediates that could not be identified in 1D NMR monitoring, and with the measurement of their half-lives. ${ }^{42,43}$ The possibility to collect reliable UF2DNMR data with a flow unit should thus provide this information in conditions that more closely replicate the reaction conditions of interest. While the analysis of more complex reactions is planned in our laboratory, it will require the installation of a ductless fume hood close to the NMR magnet. The flow-unit can be used with arbitrary magnets and $5 \mathrm{~mm}$ probes, but a limitation of the approach is that most NMR facility do not have a fume hood already installed closed to the NMR magnets.

An important feature of 2D NMR is that, in contrast to $1 D$ NMR, the proportionality factor between peak volumes and the concentrations of nuclei is peak-specific. This can be observed in Fig. 6 , where the relative intensities for the $\mathrm{CH}_{3} / \mathrm{CH}_{3} / \mathrm{CH}_{2}$ ethyl acetate peaks are not $3 / 3 / 2$. This effect is more pronounced here because of the constant time nature of the experiment: the peak integrations do not solely depend on the number of nuclei, but also on the pulse sequence parameters. In order to obtain absolute concentrations for each compound, a calibration step is needed. The calibration is in some cases possible for the initial reactants, with spectra collected before the start of the reaction (as illustrated in the ESI). In practice, in applications reported so far UF2DNMR has been used to measure half-lives, which only depend on the relative variation of the signal.
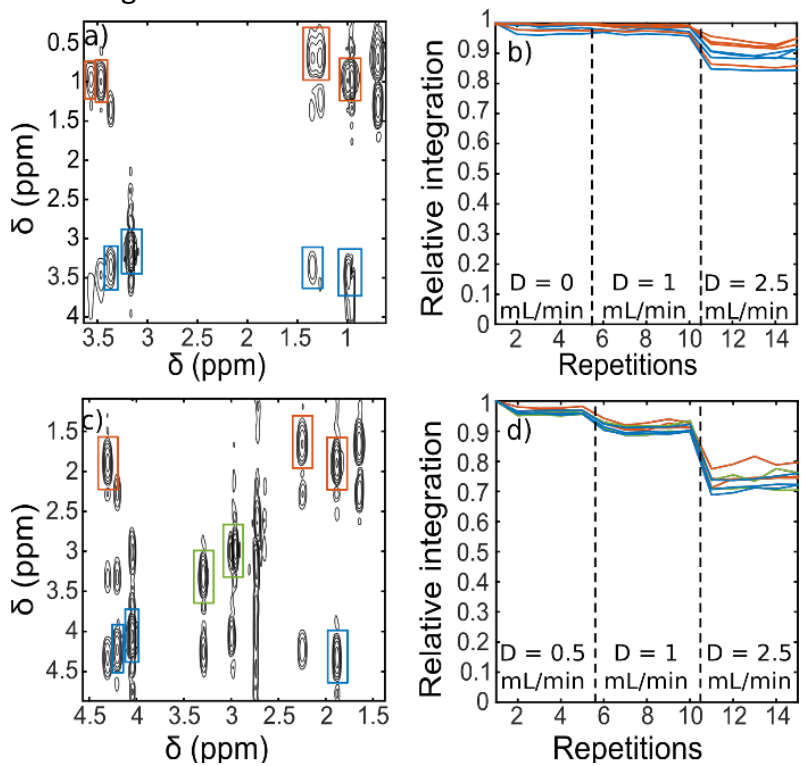

Figure 5: Ultrafast COSY spectrum obtained with spatial encoding on X-axis on mode mixture 1 in water (a) and in acetonitrile (c) and variation of peak integration as a function of repetitions at different flow rates ( $b$ and $d$ ).

Scheme 1: Saponification reaction of ethyl acetate.
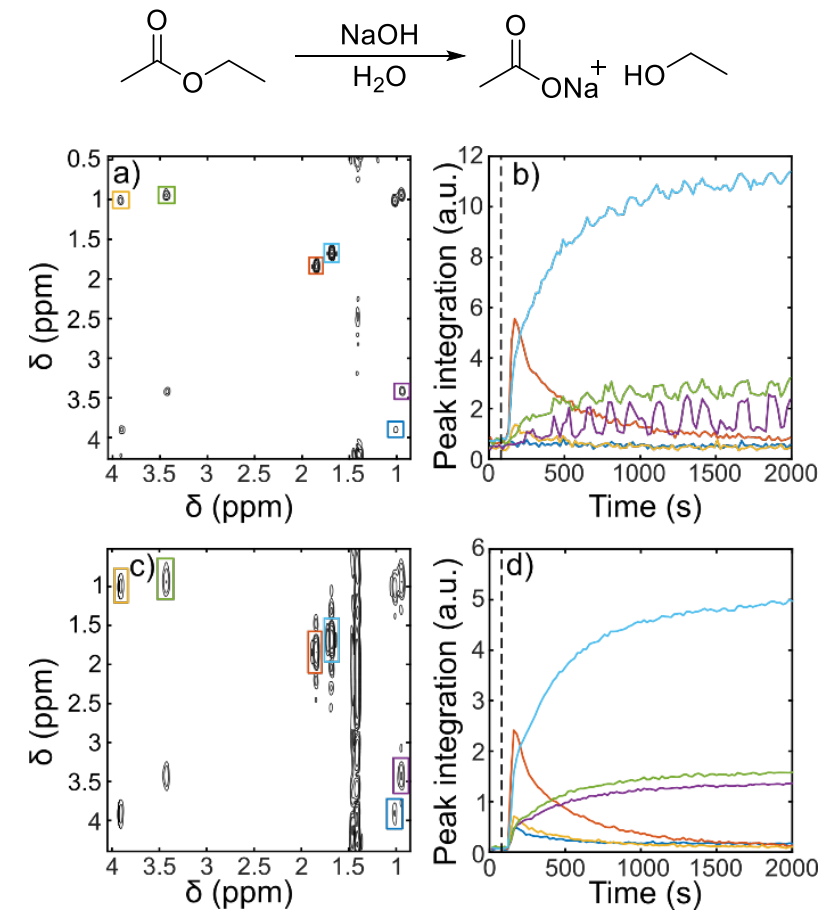

Figure 6: UFCOSY spectra with Z-axis (a) and X-axis (c) encoding and evolution of the peak volumes for selected signals ( $b$ and d) during a saponification reaction (ethyl acetate: red, yellow and blue; acetic acid: cyan; and ethanol: green and purple). UFCOSY spectra were recorded every $10 \mathrm{~s}$, alternating $\mathrm{X}$ and $\mathrm{Z}$-spatial encoding. The dashed line indicates when ethyl acetate was introduced in the reaction mixture. 


\section{CONCLUSION}

We have shown that UF2DNMR can be used for online monitoring in flow conditions, using a commercially available flow unit. Good-quality COSY spectra were obtained from solution mixtures in less than 1 second, for flow rates of up to 3 $\mathrm{mL} / \mathrm{min}$. Signal fluctuations were observed, which originate from the pulsatile nature of the flow and interferences between spatial encoding and sample motion. These fluctuations can be suppressed using transverse rather than longitudinal spatial encoding, at the cost of a loss of resolution in the indirect dimension. A model saponification reaction was monitored with a time-series of COSY spectra, acquired every $10 \mathrm{~s}$. Real-time monitoring with UF2DNMR has the potential to increase the kinetic and mechanistic information that can be accessed with online monitoring.

\section{Conflicts of interest}

There are no conflicts to declare.

\section{Acknowledgements}

The authors thank Ludmilla Guduff, Martin Hofmann and Matteo Pennestri for useful discussion and advice. This work has received funding from the European Research Council (ERC) under the European Union's Horizon 2020 research and innovation program (grant agreement No 801774), the Region Pays de la Loire (Connect Talent), and the Agence Nationale de la Recherche (ANR-16- CE29-0012).

\section{Notes and references}

1 D. G. Blackmond, J. Am. Chem. Soc., 2015, 137, 10852-10866.

2 P. Renzi, J. Hioe and R. M. Gschwind, Acc. Chem. Res., 2017, 50, 2936-2948.

3 R. Poli, Comment. Inorg. Chem., 2009, 30, 177-228.

4 D. A. Foley, A. L. Dunn and M. T. Zell, Magn. Reson. Chem., 2016, 54, 451-456.

5 L. Cerofolini, S. Giuntini, L. Barbieri, M. Pennestri, A. Codina, M. Fragai, L. Banci, E. Luchinat and E. Ravera, Biophys. J., 2019, 116, 239-247.

6 E. Danieli, J. Perlo, A. L. L. Duchateau, G. K. M. Verzijl, V. M. Litvinov, B. Blümich and F. Casanova, ChemPhysChem, 2014, 15 , 3060-3066.

7 M. Tabatabaei Anaraki, R. Dutta Majumdar, N. Wagner, R. Soong, V. Kovacevic, E. J. Reiner, S. P. Bhavsar, X. Ortiz Almirall, D. Lane, M. J. Simpson, H. Heumann, S. Schmidt and A. J. Simpson, Anal. Chem., 2018, 90, 7912-7921.

8 A. M. R. Hall, J. C. Chouler, A. Codina, P. T. Gierth, J. P. Lowe and U. Hintermair, Catal. Sci. Technol., 2016, 6, 8406-8417.

9 A. Brächer, S. Hoch, K. Albert, H. J. Kost, B. Werner, E. von Harbou and H. Hasse, J. Magn. Res., 2014, 242, 155-161.
10 M. Maiwald, H. H. Fischer, Y.-K. Kim, K. Albert and H. Hasse, J. Magn. Res., 2004, 166, 135-146.

11 E. von Harbou, R. Behrens, J. Berje, A. Brächer and H. Hasse, Chem-Ing-Tech., 2017, 89, 369-378.

12 A. Brächer, R. Behrens, E. von Harbou and H. Hasse, Chemical Engineering Journal, 2016, 306, 413-421.

13 D. A. Foley, E. Bez, A. Codina, K. L. Colson, M. Fey, R. Krull, D. Piroli, M. T. Zell and B. L. Marquez, Anal. Chem., 2014, 86, 1200812013.

14 M. Khajeh, M. A. Bernstein and G. A. Morris, Magn. Reson. Chem., 2010, 48, 516-522.

15 A. M. R. Hall, R. Broomfield-Tagg, M. Camilleri, D. R. Carbery, A. Codina, D. T. E. Whittaker, S. Coombes, J. P. Lowe and U. Hintermair, Chem. Commun., 2018, 54, 30-33.

16 A. M. R. Hall, P. Dong, A. Codina, J. P. Lowe and U. Hintermair, ACS Catal., 2019, 9, 2079-2090.

17 C. Schotten, J. L. Howard, R. L. Jenkins, A. Codina and D. L. Browne, Tetrahedron, 2018, 74, 5503-5509.

18 R. O. Kühne, T. Schaffhauser, A. Wokaun and R. R. Ernst, J. Magn. Res., 1979, 35, 39-67.

19 M. D. Christianson, E. H. P. Tan and C. R. Landis, J. Am. Chem. Soc., 2010, 132, 11461-11463.

20 A. L. Dunn and C. R. Landis, Macromolecules, 2017, 50, 22672275.

21 A. C. Jones, A. W. Sanders, M. J. Bevan and H. J. Reich, J. Am. Chem. Soc., 2007, 129, 3492-3493.

22 A. C. Jones, A. W. Sanders, W. H. Sikorski, K. L. Jansen and H. J. Reich, J. Am. Chem. Soc., 2008, 130, 6060-6061.

23 K. N. Plessel, A. C. Jones, D. J. Wherritt, R. M. Maksymowicz, EricT. Poweleit and H. J. Reich, Org. Lett., 2015, 17, 2310-2313.

24 S. E. Denmark, B. J. Williams, B. M. Eklov, S. M. Pham and G. L. Beutner, J. Org. Chem., 2010, 75, 5558-5572.

25 A. A. Thomas and S. E. Denmark, Science, 2016, 352, 329-332.

26 G. Carret, T. Berthelot and P. Berthault, Anal. Chem., 2017, 89, 2995-3000.

27 A. J. Oosthoek-de Vries, P. J. Nieuwland, J. Bart, K. Koch, J. W. G. Janssen, P. J. M. van Bentum, F. P. J. T. Rutjes, H. J. G. E. Gardeniers and A. P. M. Kentgens, J. Am. Chem. Soc., 2019, 141, 5369-5380.

28 E. Kupče, T. Nishida and R. Freeman, Prog. Nucl. Mag. Res. Sp., 2003, 42, 95-122.

29 M. W. Maciejewski, H. Z. Qui, I. Rujan, M. Mobli and J. C. Hoch, J. Magn. Res., 2009, 199, 88-93.

30 J. C. Hoch, M. W. Maciejewski, M. Mobli, A. D. Schuyler and A. S. Stern, Acc. Chem. Res., 2014, 47, 708-717.

31 M. Mobli and J. C. Hoch, Prog. Nucl. Mag. Res. Sp., 2014, 83, 21-41.

32 D. Jeannerat, in Encyclopedia of Magnetic Resonance, ed. R. K. Harris, John Wiley \& Sons, Ltd, Chichester, UK, 2011

33 G. Bodenhausen and R. R. Ernst, J. Magn. Res., 1981, 45, 367373.

34 O. Millet and M. Pons, J. Magn. Res., 1998, 131, 166-169.

35 R. Dass, W. Koźmiński and K. Kazimierczuk, Anal. Chem., 2015, 87, 1337-1343.

36 M. Urbańczyk, A. Shchukina, D. Gołowicz and K. Kazimierczuk, Magn. Reson. Chem., 2019, 57, 4-12.

37 Y. Wu, C. D'Agostino, D. J. Holland and L. F. Gladden, Chem. Commun., 2014, 50, 14137-14140.

38 G. Gasparini, B. Vitorge, P. Scrimin, D. Jeannerat and L. J. Prins, Chem. Commun., 2008, 3034.

39 L. Frydman, T. Scherf and A. Lupulescu, P. Natl. A. Sci., 2002, 99, 15858-15862. 
40 L. Frydman, A. Lupulescu and T. Scherf, J. Am. Chem. Soc., 2003, 125, 9204-9217.

41 P. Pelupessy, J. Am. Chem. Soc., 2003, 125, 12345-12350.

42 A. Herrera, E. Fernández-Valle, R. Martínez-Álvarez, D. Molero, Z. D. Pardo, E. Sáez and M. Gal, Angew. Chem., 2009, 48, 62746277.

43 A. Herrera, E. Fernández-Valle, R. Martínez-Álvarez, D. MoleroVílchez, Z. D. Pardo-Botero and E. Sáez-Barajas, Magn. Reson. Chem., 2015, 53, 952-970.

44 I. Fernández, M. E. Fernández-Valle, R. Martínez-Álvarez, D. Molero-Vílchez, Z. D. Pardo, E. Sáez-Barajas, Á. Sánchez and A. Herrera, J. Org. Chem., 2014, 79, 8086-8093.

45 M. E. Fernández-Valle, R. Martínez-Álvarez, D. Molero-Vílchez, Z. D. Pardo, E. Sáez-Barajas and A. Herrera, J. Org. Chem., 2015, 80, 799-805.

46 M. Gal, M. Mishkovsky and L. Frydman, J. Am. Chem. Soc., 2006, 128, 951-956.

47 L. H. K. Queiroz, P. Giraudeau, F. A. B. dos Santos, K. T. Oliveira and A. G. Ferreira, Magn. Reson. Chem., 2012, 50, 496-501.

48 R. Boisseau, U. Bussy, P. Giraudeau and M. Boujtita, Anal. Chem., 2015, 87, 372-375.

49 B. Shapira, A. Karton, D. Aronzon and L. Frydman, J. Am. Chem. Soc., 2004, 126, 1262-1265.

50 L. H. K. Queiroz Júnior, D. P. K. Queiroz, L. Dhooghe, A. G. Ferreira and P. Giraudeau, Analyst, 2012, 137, 2357.

51 B. Gouilleux, B. Charrier, E. Danieli, J.-N. Dumez, S. Akoka, F.-X. Felpin, M. Rodriguez-Zubiri and P. Giraudeau, Analyst, 2015, 140, 7854-7858.

52 P. Giraudeau and F.-X. Felpin, React. Chem. Eng., 2018, 3, 399413.

53 B. Gouilleux, B. Charrier, S. Akoka, F.-X. Felpin, M. RodriguezZubiri and P. Giraudeau, TrAC-Trend. Anal. Chem., 2016, 83, 6575.

54 P. Mansfield, Magn. Reson. Med., 1984, 1, 370-386.

55 US. Pat., 4973 906, 1992.

56 A. Caprihan and E. Fukushima, Phys. Rep., 1990, 198, 195-235.

57 J. M. Pope and S. Yao, Concepts Magn. Reson., 1993, 5, 281302.

58 T. L. Hwang and A. J. Shaka, J. Magn. Res. Ser. A, 1995, 112, 275-279.

59 P. T. Callaghan, Principles of Nuclear Magnetic Resonance Microscopy, Clarendon Press, 1993.

60 M. A. Connell, P. J. Bowyer, P. Adam Bone, A. L. Davis, A. G. Swanson, M. Nilsson and G. A. Morris, J. Magn. Res., 2009, 198, 121-131.

61 P. Lanzer, C. Barta, E. H. Botvinick, H. U. Wiesendanger, G. Modin and C. B. Higgins, Radiology, 1985, 155, 681-686.

62 Y. Shrot, A. Tal and L. Frydman, Magn. Reson. Chem., 2009, 47, 415-422.

63 Y. Shrot and L. Frydman, J. Chem. Phys., 2009, 131, 224516.

64 R. J. Ogg, R. B. Kingsley and J. S. Taylor, J. Magn. Res. Ser. B, 1994, 104, 1-10. 